\title{
A standardized extract of cultured Lentinula edodes mycelia downregulates cortactin in gemcitabine-resistant pancreatic cancer cells
}

\author{
SHAJEDUL ISLAM ${ }^{1,2}$, TAKAO KITAGAWA ${ }^{1}$, BYRON BARON ${ }^{3}$, KEISUKE KUHARA ${ }^{4}$, \\ HIROKI NAGAYASU ${ }^{4}$, MASANOBU KOBAYASHI ${ }^{1}$, ITSUO CHIBA ${ }^{5}$ and YASUHIRO KURAMITSU ${ }^{1}$ \\ ${ }^{1}$ Advanced Research Promotion Center, Health Sciences University of Hokkaido, Ishikari-Tobetsu, \\ Hokkaido 061-0293; ${ }^{2}$ Oral Health Science Center, Tokyo Dental College, Chiyoda, Tokyo 101-0061, Japan; \\ ${ }^{3}$ Faculty of Medicine and Surgery, Centre for Molecular Medicine and Biobanking, University of Malta, Msida, \\ MSD 2080, Malta; ${ }^{4}$ Division of Oral and Maxillofacial Surgery, Department of Human Biology and Pathophysiology, \\ School of Dentistry, Health Sciences University of Hokkaido; ${ }^{5}$ Division of Disease Control and Molecular Epidemiology, \\ Department of Oral Growth and Development, School of Dentistry, \\ Health Sciences University of Hokkaido, Ishikari-Tobetsu, Hokkaido 061-0293, Japan
}

Received March 1, 2021; Accepted June 21, 2021

DOI: $10.3892 / \mathrm{ol} .2021 .12915$

\begin{abstract}
AHCC}^{\circledR}$, a standardized extract of cultured Lentinula edodes mycelia, enhances the therapeutic effects and reduces the adverse effects of chemotherapy. Our previous study reported that treatment with $\mathrm{AHCC}^{\circledR}$ downregulated the expression levels of tumor-associated proteins in the gemcitabine-resistant pancreatic cancer cell line, KLM1-R. However, to the best of our knowledge, the role of $\mathrm{AHCC}^{\circledR}$ in the inhibition of cell migration remains unexplored. Cortactin (CTTN), an actin nucleation-promoting factor, has been reported to be upregulated and correlated with migration, invasion and metastasis in pancreatic cancer cells. The present study aimed to investigate the effects of $\mathrm{AHCC}^{\circledR}$ on cell migration and the protein expression level of CTTN in KLM1-R cells. The Gene Expression Profiling Interactive Analysis (GEPIA2), an online bioinformatics platform, was used to analyze CTTN mRNA expression levels in pancreatic cancer tissues compared with normal pancreatic tissues. CTTN mRNA expression and its association with clinicopathological characteristics were assessed by using the GEPIA2 platform. Next, the effects of $\mathrm{AHCC}^{\circledR}$ on KLM1-R cell migration were investigated by in vitro wound-healing assay. The KLM1-R cells were treated with $\mathrm{AHCC}^{\circledR}$ at a concentration of $10 \mathrm{mg} / \mathrm{ml}$ for $48 \mathrm{~h}$. Western blotting was performed on of cell lysates with anti-CTTN or anti-actin antibodies to
\end{abstract}

Correspondence to: Professor Yasuhiro Kuramitsu, Advanced Research Promotion Center, Health Sciences University of Hokkaido, 1757 Kanazawa, Ishikari-Tobetsu, Hokkaido 061-0293, Japan

E-mail: climates@hoku-iryo-u.ac.jp

Key words: $\mathrm{AHCC}^{\circledast}$, pancreatic cancer, KLM1-R, cortactin, migration assess the protein expression levels of CTTN. Bioinformatics analysis indicated that the mRNA expression level of CTTN increased in pancreatic cancer tissues. The increased mRNA expression levels of CTTN were inversely associated with clinicopathological characteristics, including disease stages and prolonged patient survival times. The administration of $10 \mathrm{mg} / \mathrm{ml} \mathrm{AHCC}{ }^{\circledR}$ significantly inhibited KLM1-R cells migration compared with controls. The protein expression levels of CTTN were significantly reduced in $\mathrm{AHCC}^{\circledR}$-treated KLM1-R cells, whereas actin expression was not affected. The downregulation of CTTN indicated the anti-metastatic potential of $\mathrm{AHCC}^{\circledR}$ in pancreatic cancer cells. Overall, $\mathrm{AHCC}^{\circledR}$ may have the potential to be a complementary and alternative therapeutic approach in treating pancreatic cancer.

\section{Introduction}

Pancreatic cancer is one of the most common human malignancies and a leading cause of cancer-related mortality worldwide. Patients with pancreatic cancer often have a poor prognosis. Despite advances in oncology, the overall 5-year survival rates of this cancer have not significantly improved for decades (1). The lack of adequate and credible interventions is the key cause of the high mortality rate in pancreatic cancer. Gemcitabine is the most effective and extensively used chemotherapeutic in pancreatic cancer thus far. However, the overall survival is dismal and is in part caused by gemcitabine resistance in pancreatic cancer (2). New therapeutic agents for the treatment of pancreatic cancer are thus urgently needed.

The metastatic progression of cancer cells requires the remodeling of the actin cytoskeleton. The altered expression of key regulatory proteins of the actin cytoskeleton, such as cortactin (CTTN), contributes to carcinogenesis (3). CTTN is involved in various cell functions, including actin polymerization, the formation of cell motility structures such 
as podosomes and invadopodia, and extracellular matrix (ECM)-protein deposition. These functions of CTTN can lead to deregulated cell migration, invasion, and metastasis (4).

$\mathrm{AHCC}^{\circledast}$ is a standardized extract of Lentinula edodes mycelia. $\mathrm{AHCC}^{\circledR}$ comprises polysaccharides, amino acids, minerals, and lipids enriched in a 1,4-glucans (5). The anti-oxidant, anti-tumor, and immunomodulatory potentials of $\mathrm{AHCC}^{\circledR}$ were described in several studies $(6,7)$. Previously we reported that $\mathrm{AHCC}^{\circledR}$ downregulated tumor-associated proteins involved in pancreatic carcinogenesis (8-11). We demonstrated that following administration of $\mathrm{AHCC}^{\circledR}$, the level of heat-shock protein 27 (HSP27), heat shock factor 1 (HSF1), sex-determining region Y-box 2 (SOX-2), and CUB domain-containing protein 1 (CDCP1) were significantly reduced in pancreatic cancer cells. Moreover, our proteomic analysis revealed that HSP27 expression was strongly related to gemcitabine resistance in pancreatic cancer (12). Similarly, SOX-2 and CDCP1 are highly expressed in malignant tissues and involved in tumor invasion and metastasis $(8,11)$. Together, these findings suggested that $\mathrm{AHCC}^{\circledR}$ might suppress the proteins involved in chemoresistance and malignant progression of pancreatic cancer. Therefore, we hypothesized that $\mathrm{AHCC}^{\circledR}$ might inhibit CTTN expression and have an anti-metastatic potential in pancreatic carcinogenesis.

In the present study, we investigated CTTN mRNA expression levels in pancreatic cancer tissues from The Cancer Genome Atlas (TCGA) databases using an online bioinformatics platform. Next, we examined whether $\mathrm{AHCC}^{\circledR}$ suppressed cell migration and CTTN expression in KLM1-R cells using an in-vitro wound-healing assay and western blotting, respectively.

\section{Materials and methods}

mRNA expression analysis of CTTN in pancreatic cancer patients. The CTTN mRNA expression in pancreatic cancer tissues was analyzed from the TCGA and GTEx databases, using the Gene Expression Profiling Interactive Analysis (GEPIA2) platform (13). The GEPIA2 platform was also utilized to perform the association analyses of the CTTN expression level in pancreatic cancer with clinical characteristics, including pathological stages and Kaplan-Meier survival plots. The mRNA expression cut-off criteria were selected as follows: $\operatorname{LogFC}$ cut-off $=1$; P-value cut-off $=0.05$; datasets=pancreatic ductal adenocarcinoma; and matched normal data=match TCGA normal and GTEx. The quartile cut-off was selected for survival plot analysis. $\mathrm{P}<0.05$ is considered to indicate a statistically significant difference.

Cancer cell line and conditions. The KLM1-R pancreatic cancer cell line is gemcitabine-resistant. It has been established at the Department of Surgery and Science, Kyushu University Graduate School of Medical Science, and derived from the gemcitabine-sensitive pancreatic cancer cell line KLM1, that was exposed to gemcitabine. In brief, the KLM1 cells cultured at an initial density of $1 \times 10^{6}$ cells on 6 -well flat-bottomed plates containing $2 \mathrm{ml}$ medium for 1 day were treated with $10 \mu \mathrm{g} / \mathrm{ml}$ gemcitabine for 1 week. Cells were then cultured in a gemcitabine-free medium for 2 weeks to recover cell density. After repeating the above treatment $4 x$, a gemcitabine-resistant cell line, KLM1-R was established. KLM1-R exhibited stable characteristics with respect to growth rate, morphology, and drug resistance (14). The cells were then kept in RPMI-1640 medium supplemented with $10 \%$ fetal bovine serum (inactivated at $56^{\circ} \mathrm{C}$ for $30 \mathrm{~min}$ ), $2 \mathrm{mM}$ L-glutamine, $1.5 \mathrm{~g} / 1$ sodium bicarbonate, $10 \mathrm{mM}$ $\mathrm{N}$-2-hydroxyehylpiperazine-N'-2-ethanesulfonic acid (HEPES), and $1.0 \mathrm{mM}$ sodium pyruvate, in a $\mathrm{CO}_{2}$ incubator.

Preparation of $A H C C^{\circledast}$. $\mathrm{AHCC}^{\circledast}$ was kindly provided by Amino Up Co., Ltd. (Sapporo, Japan). AHCC ${ }^{\circledast}$ was dissolved in RPMI-1640 medium to a final concentration of $10 \mathrm{mg} / \mathrm{ml}$, filter-sterilized, and stored at $4^{\circ} \mathrm{C}$ in aliquots. Fresh $\mathrm{AHCC}^{\circledR}$ solution was used in each experiment.

In vitro wound-healing assay. The inhibition of cell migration by $\mathrm{AHCC}^{\circledast}$ was investigated by in vitro wound-healing assay as described previously (15). Briefly, cells were allowed to grow to full confluence in 24-well plates, and then a vertical wound was created with a $10 \mu 1$ pipette tip. After the cell debris was removed, a fresh RPMI-1640 complete medium supplemented $10 \%$ FBS with various concentrations of $\mathrm{AHCC}^{\circledast}[0$ (control), 1,5 and $10 \mathrm{mg} / \mathrm{ml}$ ] was added. Cells were incubated at $37^{\circ} \mathrm{C}$ and $5 \% \mathrm{CO}_{2}$ for $24 \mathrm{~h}$ and images were captured using a microscope at three time points $(3,6$, and $24 \mathrm{~h})$. Wound healing was observed at different time points within the scraped line, and representative images of scraped lines were captured using a phase-contrast microscope at $\mathrm{x} 40$ magnification. The migration area was measured and analyzed with ImageJ software (version 1.48) (16), and the experiments were conducted in triplicate.

Western blot analysis. KLM1-R cells were treated with or without $\mathrm{AHCC}^{\circledR}(10 \mathrm{mg} / \mathrm{ml})$ for $48 \mathrm{~h}$ in vitro. After treatment and washing three times, proteins were extracted from cells using lysis buffer (50 mM Tris $\mathrm{HCl}, \mathrm{pH} 7.5 ; 10 \mathrm{mM}$ EDTA, pH 7.5; $165 \mathrm{mM} \mathrm{NaCl} ; 10 \mathrm{mM} \mathrm{NaF} ; 1 \%$ Nonidet P-40; $1 \mathrm{mM}$ PMSF; $1 \mathrm{mM} \mathrm{NaVO}{ }_{3} ; 10 \mu \mathrm{g} / \mathrm{ml}$ leupeptin; and $10 \mu \mathrm{g} / \mathrm{ml}$ aprotinin). The lysis reaction was carried out for $1 \mathrm{~h}$ at $4^{\circ} \mathrm{C}$. The samples were centrifuged at $15,000 \mathrm{rpm}$ for $30 \mathrm{~min}$ at $4^{\circ} \mathrm{C}$, and the supernatant was used as a sample. Protein concentration was quantified by Lowry's protein assay. Fifteen micrograms of the protein samples were used for western blot analysis. Sodium dodecyl sulfate-polyacrylamide gel electrophoresis (SDS-PAGE) was carried out in pre-cast gels (4-20\% gradient of polyacrylamide; Mini-PROTEAN TGX Gels; Bio-Rad). After electrophoresis, gels were transferred electrophoretically onto polyvinylidene difluoride membranes (Immobilon-P; Millipore) and blocked for $1 \mathrm{~h}$ with Tris-buffered saline with $0.1 \%$ Tween-20 solution (TBS-T) containing 5\% skimmed milk. Blocked membranes were washed twice with TBS-T.

The following primary antibodies were used: Rabbit monoclonal antibody against cortactin (dilution 1:1,000, \#CST3502; Cell Signaling Technology, Beverly, MA, USA) and rabbit polyclonal antibody against actin (dilution 1:5,000, \#sc1616R; Santa Cruz Biotechnology, Inc.). Membranes were incubated with the primary antibody overnight at $4^{\circ} \mathrm{C}$, washed three times with TBS-T, and incubated with horseradish peroxidase-conjugated secondary antibody (dilution 1:10,000; Jackson Immuno-Research Laboratories Inc.) for $1 \mathrm{~h}$ at 
room temperature. Bands of cortactin and actin were visualized by the enhanced chemiluminescence system (Clarity ${ }^{\mathrm{TM}}$ Western ECL Substrate; Bio-Rad) and LuminoGraph I (ATTO Corporation, Tokyo, Japan) and recorded using ImageSaver6 software (ATTO Corporation) (17). Cortactin and actin levels were quantified by analyzing each band intensity using CS Analyzer4 software (ATTO Corporation). Each experiment was performed in triplicate. Data are expressed as mean \pm standard error (SE) of the target protein ratio to actin protein.

Literature review of the effects of $A H C C^{\circledR}$ in different cancers. Two databases, namely PubMed and Scopus, were screened for relevant articles and were limited to articles published in English. Data were extracted from the databases on May 22 , 2021, without applying any time restrictions. The formulated search strategy was used in the databases: (Active hexose correlated compound [MeSH Terms]) OR (AHCC [MeSH Terms]) AND (cancer [MeSH Terms]) OR (neoplasm [MeSH Terms]). After a comprehensive analysis, 17 studies were selected $(8-11,18-30)$

Statistical analysis. Statistical analysis was performed on a database using IBM SPSS Statistics v22 (IBM Corp.). All data are expressed as the mean \pm SE. When ANOVA indicated differences among the groups, multiple comparisons among each experimental group were performed using Bonferroni's method. Mann-Whitney U test was performed between groups of $\mathrm{AHCC}^{\circledR}$-treated and untreated samples. All statistical experiments were performed three times, and $\mathrm{P}<0.05$ was considered to indicate a statistically significant difference.

\section{Results}

CTTN mRNA expression is inversely correlated with prolonged patient survival. The CTTN mRNA expression level in pancreatic cancer tissues was analyzed using the GEPIA2 platform. The results demonstrated that the CTTN mRNA expression level was significantly increased in pancreatic cancer tissues when compared to normal pancreatic tissues (Fig. 1A). Further analysis of TCGA pancreatic cancer data in GEPIA2 showed that CTTN expression was positively correlated with the pathological disease stages, underlying their prognostic value for pancreatic cancer (Fig. 1B; P=0.012). The Kaplan-Meier survival plots demonstrated a significant relation with elevated CTTN expression in pancreatic cancer. The high level of CTTN is inversely correlated with prolonged patient survival (Fig. 1C; $\mathrm{P}=0.032$ ) and disease-free survival states in patients with pancreatic cancer (Fig. 1D; $\mathrm{P}=0.037$ ).

AHCC ${ }^{\circledR}$ inhibited migration of KLM1-R cells. The effects of $\mathrm{AHCC}^{\circledR}$ on KLM1-R cell migration were assessed by wound healing assay. As shown in Fig. 2A, photomicrographs taken $24 \mathrm{~h}$ after wounding showed delayed wound closure by KLM1-R cells treated with $\mathrm{AHCC}^{\circledR}$ at concentrations ranging from 0 to $10 \mathrm{mg} / \mathrm{ml}$. Quantification of the wound closure over time revealed a significant inhibitory effect of $\mathrm{AHCC}^{\circledR}$ on KLM1-R cell motility at the concentrations of $10 \mathrm{mg} / \mathrm{ml}$ (Fig. 2B; $\mathrm{P}=0.000$ ). Beyond these dosages [0 (control), 1, and $5 \mathrm{mg} / \mathrm{ml}$, adjacent cells migrated toward the scratched space on plates, and the gap was closed as time passed. These data indicate that $\mathrm{AHCC}^{\circledR}$ effectively inhibits the migration of KLM1-R cells.

AHCC $C^{\circledR}$ treatment decreased CTTN protein levels in KLMI-R cells. To evaluate the effect of AHCC ${ }^{\circledR}$ on the CTTN expression, we analyzed the intracellular proteins from KLM1-R cells treated with or without $\mathrm{AHCC}^{\circledR}$ by western blot analysis with primary antibodies against CTTN and actin. The protein expression of CTTN was reduced by $\mathrm{AHCC}^{\circledR}$ treatment in KLM1-R cells, whereas actin was almost the same in all cells (Fig. 3A). In addition, the ratio of intensities of CTTN/actin in KLM1-R cells was measured. The CTTN/actin intensity ratio was significantly different between $\mathrm{AHCC}^{\circledR}$-treated and untreated KLM1-R cells (Fig. 3B; $\mathrm{P}=0.049$ ). These results suggested that CTTN was down-regulated by $\mathrm{AHCC}^{\circledR-}$ treatment in vitro.

AHCC ${ }^{\circledR}$ improve treatment outcome and reduces chemotherapeutic adverse effects. The literature review was performed to investigate the effects of $\mathrm{AHCC}^{\circledR}$ in different cancers. The search strategy resulted in 48 potentially eligible studies, with 16 in PubMed and 32 in Scopus. After removing the duplicates, the first screening resulted in 37 studies singled out for evaluation. After a comprehensive analysis, 17 studies were chosen based on the criteria which were set. The effects of $\mathrm{AHCC}^{\circledR}$ and its potential clinical relevance in different cancers were explored, as shown in Table I. In brief, $\mathrm{AHCC}^{\circledR}$ induces apoptosis, inhibits cellular proliferation and malignant progression in various cancer types. The apoptotic effects of $\mathrm{AHCC}^{\circledR}$ have been described to be mediated via both intrinsic and extrinsic apoptotic mechanisms. In addition, $\mathrm{AHCC}^{\circledR}$ was shown to decrease the levels of cellular reactive oxygen species (ROS). The inhibition of ROS via administration of $\mathrm{AHCC}^{\circledR}$ has been suggested to be involved in maintaining cellular integrity and preventing ROS-induced carcinogenesis. Meanwhile, it was also found that in combination with conventional anti-tumor agents, $\mathrm{AHCC}^{\circledR}$ improves the immune system and prevents immune invasion of cancer cells. These immunomodulatory effects of $\mathrm{AHCC}^{\circledR}$ are described to be involved in prolonging patient survival and reducing chemotherapeutic-related adverse effects.

\section{Discussion}

In the present study, we demonstrated that CTTN mRNA expression was significantly higher in pancreatic cancer than in normal tissues. The higher CTTN expression was significantly correlated with the pathological stages of pancreatic cancer. The Kaplan-Meier survival plots showed that elevated CTTN expression levels are inversely associated with prolonged patient survival. From our in vitro analysis, we showed that AHCC $^{\circledR}$ significantly suppressed KLM1-R cell migration. A significant reduction of CTTN protein level was observed in cells treated with $\mathrm{AHCC}^{\circledR}$ compared to control. The downregulation of CTTN possibly causes the anti-tumor potential of $\mathrm{AHCC}^{\circledR}$ in pancreatic cancer cells.

CTTN has been documented to play essential roles in regulating actin cytoskeletal dynamics (31). The basic structure of CTTN consists of four major domains: An N-terminal acidic (NTA), a central 6.5 tandems repeat, a proline-rich 
A

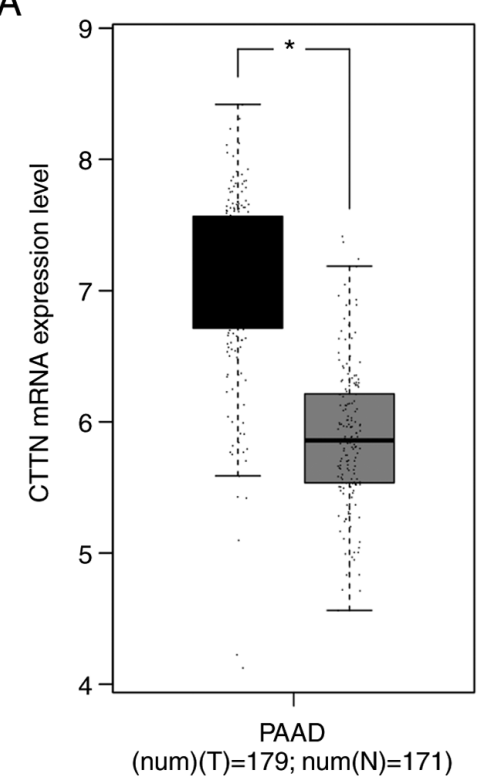

C

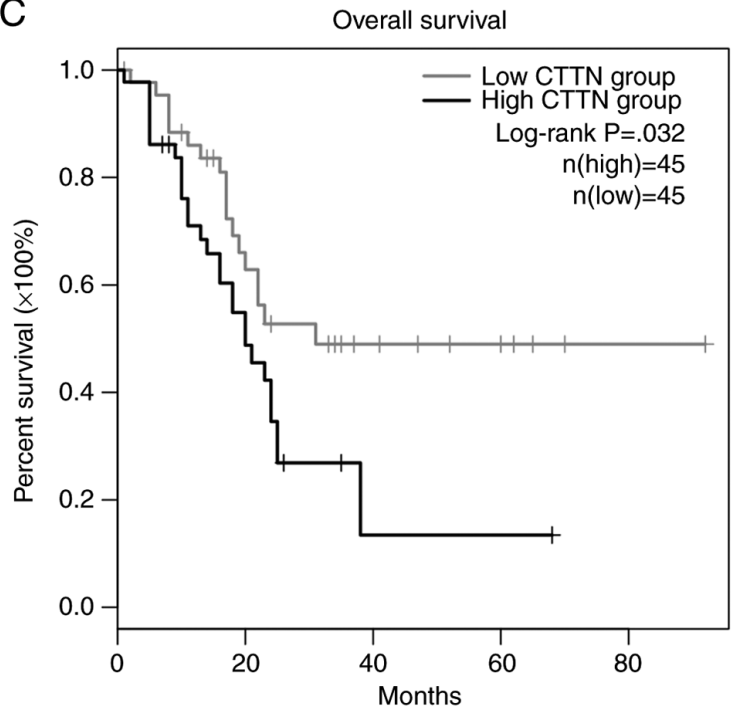

B

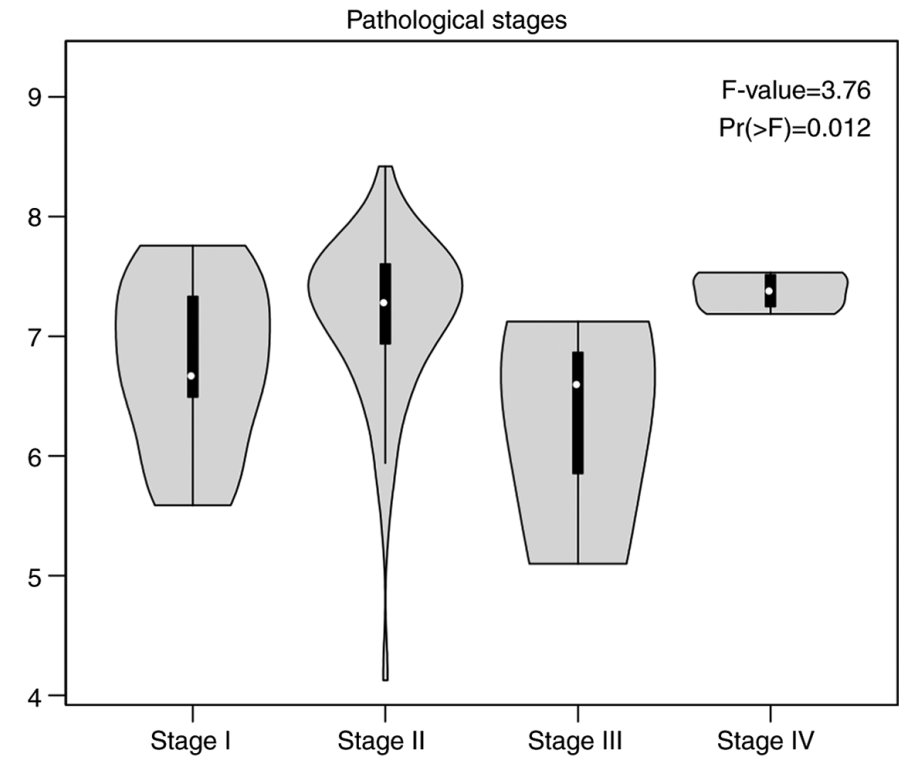

D

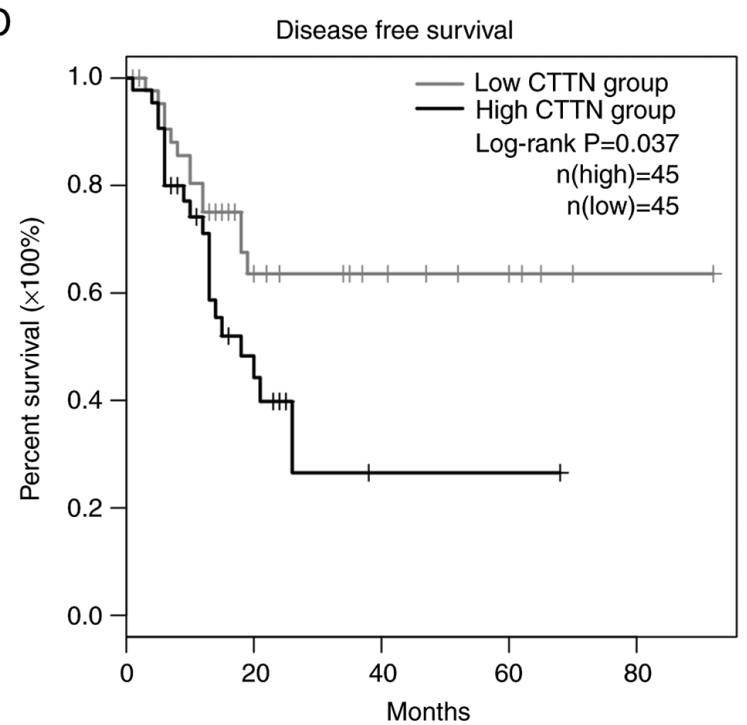

Figure 1. Expression level of CTTN mRNA and its clinical association with pancreatic cancer. (A) mRNA expression level of CTTN in pancreatic cancer tissues; boxplots were downloaded from the GEPIA2 platform. Black and gray box represent the expression levels in pancreatic cancer tissues and normal tissues, respectively. (B) Association of mRNA expression and pathological tumor stages in pancreatic cancer. Violin plots were created using GEPIA2 based on The Cancer Genome Atlas pancreatic cancer dataset. F-value indicates the statistical value of the F test; $\operatorname{Pr}(>\mathrm{F})$ indicates P-value. (C) Kaplan-Meier survival plot analysis. Overall survival analysis of CTTN performed using the GEPIA2 platform. Overall survival curve for CTTN expression in pancreatic cancer patients compared a high-risk group and a low-risk group. (D) Disease-free survival analysis of CTTN in pancreatic cancer patients comparing a high-risk group and a low-risk group. ${ }^{*} \mathrm{P}<0.05$. CTTN, cortactin; GEPIA2, Gene Expression Profiling Interactive Analysis; PAAD, pancreatic ductal adenocarcinoma.

domain, and the C-terminal Src homology 3 domains (SH3 domains) $(31,32)$. Those domains are modified and alter the interaction with binding partners to promote actin polymerization during cell motility. This then plays a central role in the formation of invadopodia, which are actin-driven protrusive structures in invasive cancer cells that degrade the ECM. The degraded ECM allows cancer cells to migrate towards the distant organs resulting in metastasis (33). The higher CTTN expression was previously described in several cancers and correlated with poor clinical outcomes in breast cancer, oral cancer, liver cancer, colon cancer, and melanoma $(4,31,33)$.

However, little is known about CTTN involvement in the tumor progression of pancreatic cancer. A previous study has shown that elevated CTTN expression in pancreatic cancer is significantly involved with metastatic compared to primary tumors (34). In addition, it was demonstrated that inhibition of CTTN expression impaired the migration and invasion potential of pancreatic cancer (34). Based on these findings, it is imperative to regulate CTTN to treat pancreatic cancer. Our study observed a significant reduction in cell migration and CTTN levels in $\mathrm{AHCC}^{\circledR}$ treated cells compared to untreated cells. The downregulation of CTTN possibly caused the reduced cell migration observed in this study. It is, however, still unknown how $\mathrm{AHCC}^{\circledR}$ downregulated CTTN levels in pancreatic cancer cells.

In vitro, CTTN is overexpressed and activated by Src-mediated tyrosine phosphorylation, which leads to increased migration of fibroblasts and endothelial 
Table I. Effects of $\mathrm{AHCC}^{\circledR}$ on different cancers.

Author (year)

Effects

Cancer types

(Refs.)

Kuhara et al, 2018

Downregulates CDCP1 levels; inhibits cell migration and

Pancreatic cancer

Suenaga et al, 2014 malignant progression

Pancreatic cancer proliferation

Tokunaga et al, 2015 Downregulates HSF1 levels

Nawata et al, 2014 Downregulates SOX-2 levels

Matsushita et al, 1998

Inhibits tumor metastasis and improves treatment outcome when combined with anti-tumor agents

Cowawintaweewat et al, Prolongs patient survival; decreases serum AST and ALT levels; increases IL-2 and neopterin levels

2006

Inhibits tumor metastasis and improves treatment outcome when combined with anti-tumor agents

Hangai et al, 2013 Inhibits chemotherapy-related adverse effects; inhibits cellular inflammation

Ito et al, 2014

Decreases the salivary level of HHV-6 following chemo therapy; reduces chemotherapeutical-related adverse effects

Ignacio et al, 2015

Maintains cellular ROS level and increases antioxidant production; inhibits tumor-related cytokine production

Cao et al, 2015 Induces apoptosis and inhibition of cellular proliferation

Yanagimoto et al, 2016

Reduces chemotherapy-related adverse effects; Reduces CRP and albumin levels; Improves patient outcomes

Graham et al, 2017

Inhibits cellular proliferation and migration; upregulates tumor suppressor protein miR-335 and contributes to prevent immune invasion

Fatehchand et al, 2017

Induces apoptosis through involvement with both extrinsic and intrinsic mechanisms

Choi et al, 2018

Induces apoptosis and proliferation; inhibits STAT3 phosphorylation; induces SHP-1 and inhibits cyclin D1, Bcl-2, Mcl-1, survivin and VEGF levels

Suknikhom et al, 2017

Increases $\mathrm{CD}+\mathrm{T}$ cell population and improves patient

outcome

Pancreatic cancer

Pancreatic cancer

Adenocarcinoma

Hepatocellular carcinoma

Ovarian cancer

Breast cancer

Colon, pancreatic, lung

and ovarian cancer

B6 melanoma murine

model

Hepatoma tumor-bearing

mice

Pancreatic cancer

Breast cancer

Acute myeloblastic

leukemia

Ovarian cancer

Ovarian cancer

AST, aspartate aminotransferase; ALT, alanine aminotransferase; ROS, reactive oxygen species; HSP27, heat shock protein 27; SOX-2, sex-determining region Y box 2; HHV-6, human herpesvirus 6; HSF1, heat shock factor 1; CRP, c-reactive protein; CDCP1, CUB domain-containing protein 1; STAT3, signal transducer and activator of transcription 3; Bcl-2, B cell lymphoma 2; VEGF, vascular endothelial growth factor; VE, vascular endothelial; miR, microRNA; SHP-1, tyrosine-protein phosphatase non-receptor type 6; Mcl-1, induced myeloid leukemia cell differentiation protein; $\mathrm{CD}+\mathrm{T}$, cluster of differentiation positive $\mathrm{T}$ cells.

cells $(35,36)$. Phosphorylation of CTTN occurring primarily at tyrosine 421 (Tyr421) enhances the actin assembly during cytoskeletal remodeling $(37,38)$. The tyrosine phosphorylation of CTTN correlates with the invadopodia activity necessary for matrix degradation, cell migration, and invasion (39). The protein-tyrosine phosphatase SHP-1 is a negative regulator of multiple signal transduction pathways and proposed to be a candidate tumor suppressor gene in various cancers (40). The substrates that are efficiently phosphorylated by Src kinase are, in turn, efficient substrates for SHP-1. Consequently, SHP-1 can negatively regulate Src-mediated autophosphorylation (41). Notably, a previous study has demonstrated that treatment with AHCC $^{\circledR}$ significantly elevated SHP-1 in ovarian cancer, whereas elevated SHP-1 contributed to preventing the malignant progression of this cancer (29). Since Src-kinase-mediated phosphorylation is essential for the biological relevance of CTTN in cancer progression, the elevated SHP-1 level via AHCC ${ }^{\circledR}$ may control the expression and activation of CTTN in pancreatic cancer. The other possible mechanisms cannot be ruled out. Further studies are needed to clarify our speculation and elucidate the anti-tumor potential of $\mathrm{AHCC}^{\circledR}$ in pancreatic cancer.

Meanwhile, to explore potential anti-tumor effects of $\mathrm{AHCC}^{\circledR}$ in other cancers, we performed a literature review by using effective search engines. We found that $\mathrm{AHCC}^{\circledR}$ can inhibit malignant progression by inducing cellular apoptosis and inhibition of cellular proliferation. Moreover, $\mathrm{AHCC}^{\circledR}$ decreases the cellular ROS levels and maintains endothelial 
A

$\mathrm{Oh}$
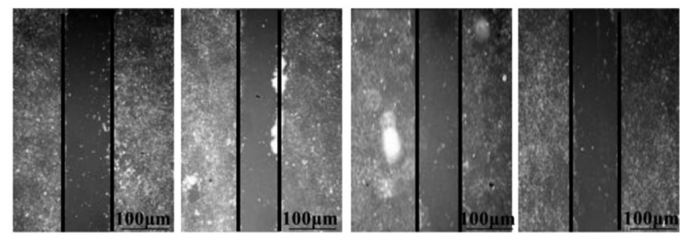

$3 \mathrm{~h}$
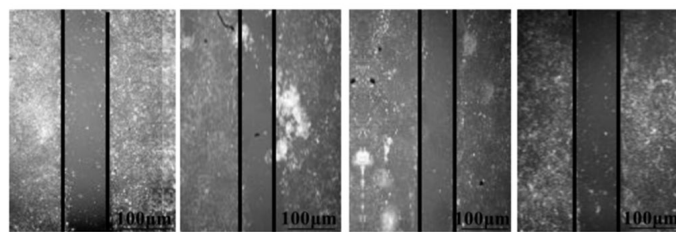

$6 \mathrm{~h}$
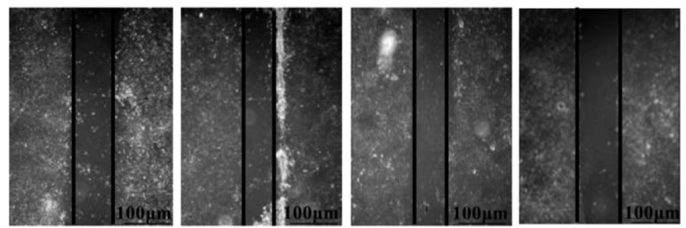

$24 \mathrm{~h}$
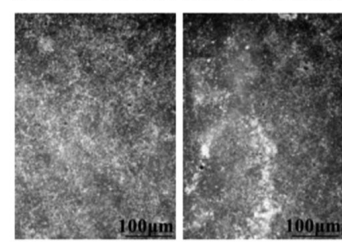

0

1

(2)

$\operatorname{AHCC}^{\circledR}(\mathrm{mg} / \mathrm{ml})$

\section{B}

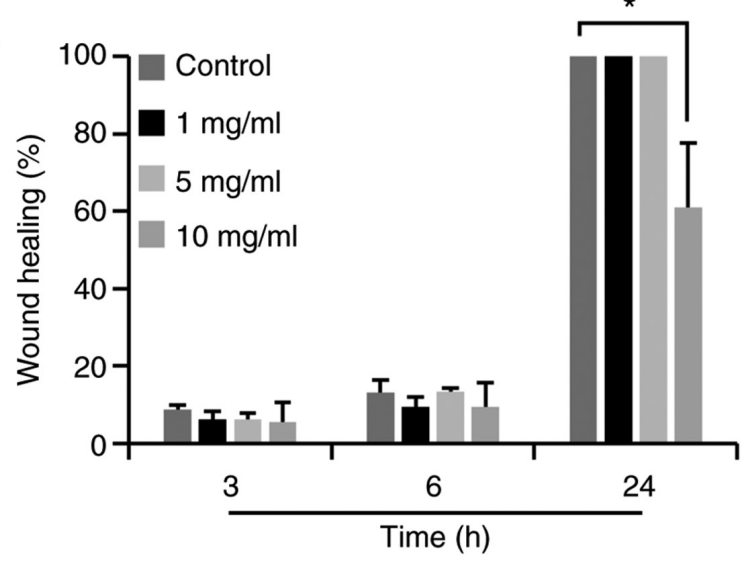

Figure 2. Effects of $\mathrm{AHCC}^{\circledR}$ on the migration capabilities in the gemcitabine-resistant pancreatic cancer cell, KLM-1R. (A) Wound healing assay was used to examine cellular migration. KLM-1R cells were allowed to grow to full confluence ( $80 \%$ ) in 24 -well plates, and then a wound was created with a sterile pipette tip. $\mathrm{AHCC}^{\circledR}$ was added to the well, and phase-contrast images of the wound at three different locations were captured at $0,3,6$ and $24 \mathrm{~h}$ (scale bar, $100 \mu \mathrm{m}$ ). Black lines represent the width of the wound. A total of three independent experiments were examined, and representative images are presented. (B) Quantification of the average wound healing degree of KLM-1R cells. Wound healing was quantified as a percentage as the mean \pm standard error. Each experiment was performed in triplicate. ${ }^{*} \mathrm{P}<0.05$.

cellular plasticity, and prevents malignant transformation. Importantly, we observed that administration of $\mathrm{AHCC}^{\circledR}$ involved immune-modulatory functions, which contribute to improving overall patient survival and reducing the adverse effects of chemotherapeutics. Collectively, these findings indicated that $\mathrm{AHCC}^{\circledast}$ plays pleiotropic roles in preventing the onset of different cancers and may constitute an attractive therapeutic agent.

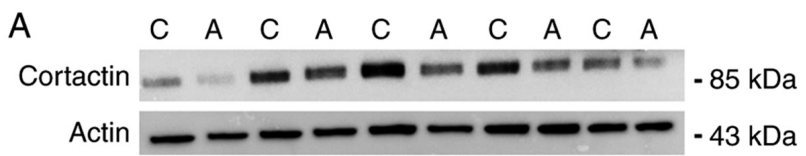

B

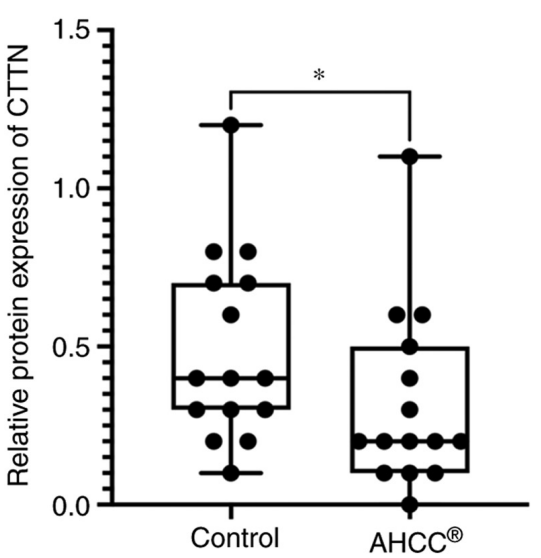

Figure 3. Effects of $\mathrm{AHCC}^{\circledR}$ on CTTN protein expression level. KLM-1R cells were treated with $\mathrm{AHCC}^{\circledR}$ at a concentration of $10 \mathrm{mg} / \mathrm{ml}$. (A) CTTN expression was analyzed by western blotting $(n=5)$. Protein expression level of CTTN was reduced in $\mathrm{AHCC}^{\circledR}$-treated KLM1-R cells, whereas actin was almost the same in all cells. (B) Ratio of intensities of CTTN/actin in KLM-1R cells was measured by using densitometry. AHCC ${ }^{\circledR}$ significantly reduced the protein level of CTTN compared with that of the untreated control ( $\left.{ }^{*} \mathrm{P}=0.049\right)$. C, control; A, AHCC ${ }^{\circledR}$. CTTN, cortactin.

In conclusion, our results suggested that $\mathrm{AHCC}^{\circledR}$ has anti-metastatic effects in pancreatic cancer cell lines via the downregulated CTTN level, and thus, this compound exhibits for the treatment of pancreatic cancer. However, a lack of adequate validation of the bioinformatics results in tissue samples is a limitation of this study. Therefore, further studies are needed to develop $\mathrm{AHCC}^{\circledR}$ as a complementary and alternative therapeutic approach to treating pancreatic cancer.

\section{Acknowledgements}

The authors would like to thank Dr Shin-ichiro Maehara and Professor Yoshihiko Maehara at Kyushu University (Fukuoka, Japan) for providing the KLM1-R cells.

\section{Funding}

This work was supported in part by Grants-in-Aid from the Ministry of Education, Science, Sports and Culture of Japan (grant no. 17K07218).

\section{Availability of data and materials}

All data generated or analyzed during this study are included in this published article.

\section{Authors' contributions}

SI, TK and YK conceived and designed the study and performed the experiments. SI, BB, KK, HN, MK, IC and YK analyzed and interpreted the data. SI wrote the initial draft of the manuscript. BB, TK and YK contributed to critical revision of the manuscript. All authors read and approved the final 
manuscript. SI and YK confirm the authenticity of all the raw data.

\section{Ethics approval and consent to participate}

Not applicable.

\section{Patient consent to participate}

Not applicable.

\section{Competing interests}

The authors declare that they have no competing interests.

\section{References}

1. Islam S, Kitagawa T, Baron B, Abiko Y, Chiba I and Kuramitsu Y: ITGA2, LAMB3, and LAMC2 may be the potential therapeutic targets in pancreatic ductal adenocarcinoma: An integrated bioinformatics analysis. Sci Rep 11: 10563, 2021.

2. Amrutkar M and Gladhaug IP: Pancreatic cancer chemoresistance to gemcitabine. Cancers (Basel) 9: 157, 2017.

3. Yamaguchi $\mathrm{H}$ and Condeelis J: Regulation of the cytoskeleton in cancer cell migration and invation. Biochim Biophys Acta 1773: 642-652, 2007.

4. Yin M, Ma W and An L: Cortactin in cancer cell migration and invasion. Oncotarget 8: 88232-88243, 2017.

5. Kamiyama Y, Matsui Y, Kawaguchi Y, Kosuna K and Wakame K: Active hexose correlated compound (AHCC). Biotherapy 14: 959-964, 2000 .

6 . Kidd P: The use of mushroom glucans and proteoglycans in cancer treatment. Altern Med Rev 5: 4-27, 2000.

7. Ye SF, Ichimura K, Wakame K and Ohe M: Suppressive effects of active hexose correlated compound on the increased activity of hepatic and renal ornithine decarboxylase induced by oxidative stress. Life Sci 74: 593-602, 2003.

8. Kuhara K, Tokuda K, Kitagawa T, Baron B, Tokunaga M, Harada K, Terasaki M, Uehara O, Ohta T, Takai R, et al: CUB domain-containing protein 1 (CDCP1) is down-regulated by active hexose-correlated compound in human pancreatic cancer cells. Anticancer Res 38: 6107-6111, 2018.

9. Suenaga S, Kuramitsu Y, Kaino S, Maehara S-I, Maehara Y, Sakaida I and Nakamura K: Active hexose-correlated compound down-regulates HSP27 of pancreatic cancer cells, and helps the cytotoxic effect of gemcitabine. Anticancer Res 34: 141-146, 2014

10. Tokunaga M, Baron B, Kitagawa T, Tokuda K and Kuramitsu Y: Active hexose-correlated compound down-regulates heat shock factor 1, a transcription factor for HSP27, in gemcitabine-resistant human pancreatic cancer cells. Anticancer Res 35: 6063-6067, 2015.

11. Nawata J, Kuramitsu Y, Wang Y, Kitagawa T, Tokuda K, Baron B, Akada J, Suenaga S, Kaino S, Maehara S, et al: Active hexose-correlated compound down-regulates sex-determining region Y-box 2 of pancreatic cancer cells. Anticancer Res 34: 4807-4811, 2014

12. Mori-Iwamoto S, Kuramitsu Y, Ryozawa S, Mikuria K, Fujimoto M, Maehara S, Maehara Y, Okita K, Nakamura K and Sakaida I: Proteomics finding heat shock protein 27 as a biomarker for resistance of pancreatic cancer cells to gemcitabine. Int J Oncol 31: 1345-1350, 2007.

13. Tang Z, Kang B, Li C, Chen T and Zhang Z: GEPIA2: An enhanced web server for large-scale expression profiling and interactive analysis. Nucleic Acids Res 47: W556-W560, 2019.

14. Maehara S, Tanaka S, Shimada M, Shirabe K, Saito Y, Takahashi K and Maehara Y: Selenoprotein P, as a predictor for evaluating gemcitabine resistance in human pancreatic cancer cells. Int J Cancer 112: 184-189, 2004.

15. Belkourchia F and Desrosiers R: The enzyme L-isoaspartyl (D-aspartyl) methyltransferase promotes migration and invasion in human U-87 MG and U-251 MG glioblastoma cell lines. Biomed Pharmacother 140: 111766, 2021

16. Schneider CA, Rasband WS and Eliceiri KW: NIH image to ImageJ: 25 years of image analysis. Nat Methods 9: 671-675, 2012.
17. Islam S, Uehara O, Matsuoka H, Kuramitsu Y, Adhikari BR, Hiraki D, Toraya S, Jayawardena A, Saito I, Muthumala M, et al: DNA hypermethylation of sirtuin 1 (SIRT1) caused by betel quid chewing-a possible predictive biomarker for malignant transformation. Clin Epigenetics 12: 12, 2020.

18. Matsushita K, Kuramitsu Y, Ohiro Y, Obara M, Kobayashi M, Li YQ and Hosokawa M: Combination therapy of active hexose correlated compound plus UFT significantly reduces the metastasis of rat mammary adenocarcinoma. Anticancer Drugs 9: 343-350, 1998.

19. Cowawintaweewat S, Manoromana S, Sriplung H, Khuhaprema T, Tongtawe P, Tapchaisri P and Chaicumpa W: Prognostic improvement of patients with advanced liver cancer after active hexose correlated compound (AHCC) treatment. Asian Pac J Allergy Immunol 24: 33-45, 2006

20. Hunter RJ, Fujii H, Wakame K, Gaikwad A, Wolf JK and Smith JA: Evaluation of active hexose correlated compound (AHCC) in combination with pegylated liposomal doxorubicin for treatment of ovarian cancer. Int J Appl Res Nat Prod 4: 6-11, 2011

21. Hangai S, Iwase S, Kawaguchi T, Kogure Y, Miyaji T, Matsunaga T, Nagumo Y and Yamaguchi T: Effect of active hexose-correlated compound in women receiving adjuvant chemotherapy for breast cancer: A retrospective study. J Altern Complement Med 19: 905-910, 2013

22. Haidari M, Zhang W and Wakame K: Disruption of endothelial adherens junction by invasive breast cancer cells is mediated by reactive oxygen species and is attenuated by AHCC. Life Sci 93: 994-1003, 2013.

23. Ito T, Urushima H, Sakaue M, Yukawa S, Honda H, Hirai K, Igura T, Hayashi N, Maeda K, Kitagawa T and Kondo K: Reduction of adverse effects by a mushroom product, active hexose correlated compound (AHCC) in patients with advanced cancer during chemotherapy-the significance of the levels of HHV-6 DNA in saliva as a surrogate biomarker during chemotherapy. Nutr Cancer 66: 377-382, 2014.

24. Ignacio RM, Kim CS, Kim YD, Lee HM, Qi XF and Kim SK: Therapeutic effect of active hexose-correlated compound (AHCC) combined with CpG-ODN (oligodeoxynucleotide) in B16 melanoma murine model. Cytokine 76: 131-137, 2015.

25. Cao Z, Chen X, Lan L, Zhang Z, Du J and Liao L: Active hexose correlated compound potentiates the antitumor effects of low-dose 5 -fluorouracil through modulation of immune function in hepatoma 22 tumor-bearing mice. Nutr Res Pract 9: 129-136, 2015.

26. Yanagimoto H, Satoi S, Yamamoto T, Hirooka S, Yamaki S, Kotsuka M, Ryota H, Michiura T, Inoue K, Matsui Y, et al: Alleviating effect of active hexose correlated compound (AHCC) on chemotherapy-related adverse events in patients with unresectable pancreatic ductal adenocarcinoma. Nutr Cancer 68: 234-240, 2016.

27. Graham ÉA, Mallet JF, Jambi M, Nishioka H, Homma K and Matar C: MicroRNA signature in the chemoprevention of functionally-enriched stem and progenitor pools (FESPP) by active hexose correlated compound (AHCC). Cancer Biol Ther 18: 765-774, 2017.

28. Fatehchand K, Santhanam R, Shen B, Erickson EL, Gautam S, Elavazhagan S, Mo X, Belay T, Tridandapani $\mathrm{S}$ and Butchar JP: Active hexose-correlated compound enhances extrinsic-pathway-mediated apoptosis of acute myeloid leukemic cells. PLoS One 12: e0181729, 2017.

29. Choi JY, Lee S, Yun SM, Suh DH, Kim K, No JH, Jeong EH and Kim YB: Active hexose correlated compound (AHCC) inhibits the proliferation of ovarian cancer cells by suppressing signal transducer and activator of transcription 3 (STAT3) activation. Nutr Cancer 70: 109-115, 2018

30. Suknikhom W, Lertkhachonsuk R and Manchana T: The effects of active hexose correlated compound (AHCC) on levels of CD4+ and CD8+ in patients with epithelial ovarian cancer or peritoneal cancer receiving platinum based chemotherapy. Asian Pac J Cancer Prev 18: 633-638, 2017.

31. Buday L and Downward J: Roles of cortactin in tumor pathogenesis. Biochim Biophys Acta 1775: 263-273, 2007

32. Ammer AG and Weed S: Cortactin branches out: Roles in regulating protrusive actin dynamics. Cell Motil Cytoskeleton 65: 687-707, 2008

33. He J, Xia TS and Wang S: Cortactin and tumor invasiveness. Chin J Cancer Prev Treat 22: 72-75, 80, 2015.

34. Stock K, Borrink R, Mikesch JH, Hansmeier A, Rehkämper J, Trautmann M, Wardelmann E, Hartmann W, Sperveslage J and Steinestel K: Overexpression and Tyr421-phosphorylation of cortactin is induced by three-dimensional spheroid culturing and contributes to migration and invasion of pancreatic ductal adenocarcinoma (PDAC) cells. Cancer Cell Int 19: 77, 2019. 
35. Patel AS, Schlechter GL, Wasilenko WJ and Somers KD Overexpression of EMS1/cortactin in NIH3T3 fibroblasts causes increased cell motility and invasion in vitro. Oncogene 16: 3227-3232, 1998.

36. Huang C, Liu J, Haudenschild CC and Zhan X: The role of tyrosine phosphorylation of cortactin in the locomotion of endothelial cells. J Biol Chem 273: 25770-25776, 1998.

37. Tehrani S, Tomasevic N, Weed S, Sakowicz R and Cooper J: Src phosphorylation of cortactin enhances actin assembly. Proc Natl Acad Sci USA 104: 11933-11938, 2007.

38. Head JA, Jiang D, Li M, Zorn LJ, Schaefer EM, Parsons JT and Weed SA: Cortactin tyrosine phosphorylation requires Rac1 activity and association with the cortical actin cytoskeleton. Mol Biol Cell 14: 3216-3229, 2003.
39. Bowden ET, Onikoyi E, Tidwell R, Myoui A, Yoneda T, Yamada KM and Mueller SC: Co-localization of cortactin and phosphotyrosine identifies active invadopodia in human breast cancer cells. Exp Cell Res 312: 1240-1253, 2006.

40. Wu C, Sun M,Liu L and Zhou W: The function of the protein tyrosine phosphatase SHP-1 in cancer. Gene 306: 1-12, 2003.

41. Frank C, Burkhardt C, Imhof D, Ringel J, Zschörnig O, Wieligmann K, Zacharias M and Böhmer FD: Effective dephosphorylation of Src substrates by SHP-1. J Biol Chem 279: 11375-11383, 2004. 